Cahiers $d u$ MONDE RUSSE

\section{Cahiers du monde russe}

Russie - Empire russe - Union soviétique et États indépendants

50/2-3 | 2009

L'Europe orientale, 1650-1730. Crises, conflits et renouveau

\title{
Russian Legal Culture Before and After CommunismFrances NETHERCOTT
}

, Londres - New York : Routledge, 2007, 198 p.

\section{Aurore Chaigneau}

\section{(2) OpenEdition}

\section{Journals}

Édition électronique

URL : https://journals.openedition.org/monderusse/9748

DOI : 10.4000/monderusse. 9748

ISSN : $1777-5388$

\section{Éditeur}

Éditions de l'EHESS

\section{Édition imprimée}

Date de publication : 15 septembre 2009

ISBN : 978-2-7132-2260-3

ISSN : $1252-6576$

\section{Référence électronique}

Aurore Chaigneau, "Russian Legal Culture Before and After CommunismFrances NETHERCOTT», Cahiers du monde russe [En ligne], 50/2-3 | 2009, mis en ligne le 14 janvier 2013, consulté le 03

septembre 2022. URL : http://journals.openedition.org/monderusse/9748 ; DOI : https://doi.org/ 10.4000/monderusse. 9748

Ce document a été généré automatiquement le 3 septembre 2022.

Tous droits réservés 


\section{Russian Legal Culture Before and After CommunismFrances NETHERCOTT}

, Londres - New York : Routledge, 2007, 198 p.

\section{Aurore Chaigneau}

\section{Frances NETHERCOTT, Russian Legal Culture Before and After Communism, Criminal Justice, Politics, and the Public Sphere, Londres - New York : Routledge, 2007, 198 p.}

Inscrit par l'auteur elle-même dans la lignée des études contemporaines sur le droit en Russie, le présent ouvrage propose néanmoins un éclairage original et rare sur la doctrine pénaliste russe du xIX ${ }^{\mathrm{e}}$ siècle. Le travail de Frances Nethercott est remarquable avant tout par ses recherches sur les travaux des juristes russes de la fin de l'Empire russe. Il nous dévoile les échanges, les positionnements et les oppositions doctrinales d'une frange de cette communauté juridique dans la tourmente. Un point en particulier a focalisé l'attention de l'auteur : la réforme de la procédure pénale et les enjeux politiques liés à la redéfinition des notions de crime et de peine par la doctrine pénaliste. Plus encore - et en cela le titre de l'ouvrage est peut-être trompeur -, Frances Nethercott s'est intéressée aux prises de position de Vladimir Solov'ev sur la réforme du droit pénal ainsi qu'aux réactions que le philosophe a suscitées, tant de la part des orthodoxes conservateurs que des libéraux imprégnés de la philosophie hégélienne. L'auteur part de cette œuvre pour situer les travaux d'autres pénalistes russes. Dans le dernier chapitre elle tente, en écho aux pratiques de ses collègues russes contemporains, de remettre ces travaux en perspective avec leur utilisation dans la période actuelle. Mais cette dernière entreprise, à peine échafaudée, laisse en suspens autant d'interrogations sur l'invocation de ces auteurs historiques dans les écrits d'aujourd'hui. De même, la piste du patriotisme est à peine évoquée. On ne pourra que déplorer qu'une telle analyse ne soit menée à son 
terme. En revanche, le cœur de l'étude, la doctrine savante du XIX ${ }^{e}$ siècle, bien que non exhaustive, mérite attention. Quatre angles de vue sont utilisés.

«Les cercles de la doctrine pénaliste libérale » : qui sont les auteurs de cette doctrine pénaliste russe? Dans les années 1850 se développent des cercles (krugi) de discussion au sein même des institutions impériales, en vue de les réformer. Les critiques ne sont plus l'apanage de salons privés mais sont désormais relayées dans les plus hautes instances. En 1858 est ainsi créé Le Journal du ministère de la Justice par un de ses fonctionnaires, Dmitrij Zamjatin. Rapidement, la revue devient un espace de discussion sur la réforme de la Justice. Les débats y sont publiés ainsi que des traductions de juristes étrangers influents, tel Carl Joseph Mittermaier, professeur à Heidelberg, venu enseigner un temps en Russie devant Vladimir Spasovič (1829-1907) et Nikolaj Tagancev (1843-1923).

Dans les années 1850, Konstantin Kavelin, qui tient salon chez lui où il reçoit certains collègues du ministère de l'Intérieur et d'autres réformateurs, crée également un cercle. Les « vieux libéraux » (K. Kavelin, M.M. Stasjulevič, A.N. Pypin, V. Spasovič), comme ils seront dénommés par la suite, vont créer ensemble en 1866 Vestnik Evropy, auquel vont collaborer des intellectuels de toute nature: l'historien Sergej Solov'ev (père de Vladimir), le dramaturge A.M. Ostrovskij, Ivan Turgenev, N.V. Uspenskij, le satiriste Mihail Saltykov-Ščedrin, mais aussi des juristes. La revue publie de nombreux textes de juristes de diverses administrations, des recensions, elle organise des réunions publiques avec les Sociétés juridiques de Moscou et de Saint- Pétersbourg, et devient un organe important de la pensée libérale. Dans les années 1880, une nouvelle génération de libéraux - derrière N. Tagancev, N.D. Sergeevskij, A.F. Koni, I. Fojnickij pour les pénalistes - les rejoindra avec bien d'autres.

4 Les vestniki ne cessent en effet de réclamer une réforme des institutions dans le sens d'un plus grand humanisme et d'une reconnaissance des droits et libertés individuelles pour les sujets de l'empereur. Dans les procès des années 1870, certains avocats, comme Spasovič, s'engagent dans la défense des accusés (dans « le procès des 193 » en 1877, entre autres). En 1874, V.A. Sologub est mandaté par le ministère des Affaires intérieures pour étudier le droit pénal des États occidentaux. Sur cette base sont formulées des propositions exposées publiquement par I. Fojnickij. Mais, malgré quelques succès, les réformateurs ne peuvent empêcher la montée en puissance de la contre-réforme et la mise aux pas de l'ensemble de l'appareil d'État : tribunaux, ministère, universités, etc.

Jusqu'à la fin de l'ancien régime, les libéraux peineront à se faire entendre. Ils doivent défendre leurs positions contre une gauche plus agressive, qui s'exprime dans les pages de Sovremennik et d'Otečestvennye zapiski, et une frange conservatrice portée par Russkij Vestnik et Moskovskie vedomosti. Les libéraux y sont caricaturés en bourgeois vendus à l'Occident et doivent faire face à une opposition accrue à partir de la contre-réforme de la fin des années 1870. En Russie, leurs revendications resteront le plus souvent lettre morte.

6 Pour autant, il ne faudrait pas voir dans ce courant un groupe homogène. Parmi les auteurs de Vestnik Evropy, on compte aussi bien Georgij Vasil'evič Čičerin, qui défend par exemple la propriété individuelle comme source de liberté et instrument de stabilité économique et politique, que le philosophe Vladimir Solov'ev, qui, dans les années 1890, défend une vision chrétienne de la personne humaine et de l'État comme postulat de toute réforme légitime. Il ne reconnaît le droit à la propriété et à la liberté qu'à cette condition. Pour certains vestniki, tel Koni, Solov'ev fait figure de « vieux slavophile » aux thèses peu porteuses. Mais malgré certaines conceptions très pieuses et orthodoxes, son 
engagement lors de la famine de 1891-1892, ses prises de position pour la défense des libertés individuelles, le droit à l'éducation, le droit de réunion, ou encore sa volonté de renforcer une forme de responsabilité sociale envers les personnes, le rangent du côté des libéraux de son époque. Solov'ev incarne finalement une sorte de libéralisme médian entre le libéralisme radical de Čičerin et le communautarisme slavophile, relayé à la fin du siècle par les conservateurs nationalistes auxquels il n'a eu de cesse de s'opposer. Cette double posture explique a contrario que ce personnage incarne aujourd'hui ce que l'on appelle parfois le libéralisme "à la russe » : respectueux de la personne humaine, mais dans un cadre défini par la morale chrétienne.

7 «L'appel à la réforme du droit pénal » : l'enlisement de la réforme du Code pénal de 1845 en est l'ultime illustration. Les juristes réclament une refonte de la procédure comme du système pénitentiaire, et surtout une reconsidération des peines infligées aux coupables. Ils exigent en particulier la révision des peines corporelles et l'abolition des châtiments publics rituels, pourtant conservés dans les Statuts de 1864 et le Code modifié en 1866. Ces châtiments, étudiés et décrits par N. Murav'ev dans les années 1870, sont désormais considérés comme dégradants, contraires à toute forme de civisme et inutiles au regard d'une conception nouvelle de l'appareil judiciaire - instrument de contrôle social et non plus expression d'un pouvoir absolu. Ces pratiques paysannes coutumières sont tenues pour des formes archaïques de justice, conduisant aux yeux de D. Drill, autre vestnik, à la négation de l'individu, et immorales selon les termes de Solov'ev.

8 À l'opposé, les réformateurs réclament une nouvelle hiérarchie des peines et l'instauration d'une justice qui permette une véritable réhabilitation sociale de l'individu en lui faisant prendre conscience de ses errements plutôt qu'en le brimant davantage. Ainsi s'opposent-ils par exemple au principe de "privation de droits", contraire au dessein de réinsertion de l'individu dans la société. Le nouveau projet de Code pénal, rédigé sur ces bases par Tagancev, Fojnickij, Koni et d'autres, ne sera finalement jamais adopté, et seules quelques modifications seront apportées avant son abandon définitif dans la première décennie du $\mathrm{xx}^{\mathrm{e}}$ siècle. Le même attentisme marque la réforme de l'administration pénitentiaire: des tentatives de modernisation sont faites dès 1879 par l'annonce d'une centralisation des administrations, mais ces espoirs seront déçus par manque de financement et de volonté d'aboutir.

9 «Crime et Châtiment - les théories » : comme l'a remarquablement montré Frances Nethercott, les pénalistes libéraux ont étudié de près les auteurs occidentaux. Le droit pénal connaît ainsi une rupture épistémologique en 1863 avec la publication par Spasovič d'un manuel de droit pénal qui est le premier à exprimer une posture critique vis-à-vis des institutions en place et convoque les règles occidentales en contrepoint. Jusque-là, les travaux scientifiques se présentaient plutôt sous forme d'étude historique des institutions russes. Le recours au comparatisme induit de nouvelles perspectives et ce travail vient rapidement à être perçu comme une critique du gouvernement. Corrélativement se développe une analyse économiste des réformes qui suggère un lien entre développement légal d'un État et niveau de développement économique.

Les philosophes s'étant intéressés au droit, comme Feuerbach qui a forgé les fondements de la justice criminelle en Europe, sont souvent cités par les réformateurs. Dans la littérature pénaliste, l'influence des sciences criminelles occidentales s'avère sensible. Les travaux anthropologiques de Cesare Lombroso, professeur à Turin, sont diffusés dans les années 1880, comme ceux de Gabriel Tarde, qui défend une analyse sociétale du crime. Ces théories ont en commun de ne pas faire une grande place à la volonté dans l'analyse 
de l'action du sujet. L'origine du crime est à rechercher dans d'autres facteurs, tantôt anthropomorphiques, tantôt sociaux. On en retrouvera ainsi des traces dans les travaux de Lev Petragiskij.

11 À la fin du siècle, la doctrine pénaliste s'affirme. Les auteurs se penchent sur le rôle de l'État dans la répression de la criminalité et sur la finalité de son action. L'étude de la relation entre individu et État dans le droit pénal est l'occasion de réclamer que les institutions jouent un rôle nouveau. Tagancev, et avec lui L.S. Belogric-Kotljarevskij, A.F. Kistjakovskij ou encore Sergeevskij, vont reprendre toute l'argumentation sociologique déployée avant eux pour demander une réforme du droit pénal prenant mieux en compte les facteurs qui poussent l'individu au crime: "La logique des châtiments a des buts différents selon les époques et doit évoluer avec le développement de l'État [...] Ce n'est pas le crime que l'on juge mais le criminel avec ses caractéristiques antisociales. Le crime commis peut légalement donner lieu à un jugement et à une peine, mais la nature et le degré de gravité de la peine doivent être déterminés par l'ensemble des particularités du criminel lui-même » (p. 53). Progressivement naît ainsi un courant se revendiquant comme "positiviste légaliste », dans la lignée de Franz von Liszt. Ce courant conduit les libéraux réformateurs autour de Fojnickij à concevoir une théorie renouvelée du crime et de la peine, dans laquelle l'État doit punir en vue d'instaurer un nouveau pacte social plus respectueux de ses sujets. Dans la doctrine pénale, cela revient à défendre l'idée selon laquelle l'État n'est pas là pour punir, mais pour aider le criminel à s'amender et à réintégrer la société.

12 «Droit et morale »: Vladimir Solov'ev incarne aujourd'hui l'une des figures centrales de cette doctrine engagée du XIX ${ }^{e}$ siècle, qui défendit avec conviction une réforme de la justice et des institutions, bataillant sans compromission contre le pouvoir autocrate réactionnaire. Pourtant son œuvre ne semble pas avoir trouvé un écho aussi large au sein de la communauté juridique russe de l'époque. À sa droite, ses travaux sont critiqués par les conservateurs opposés aux réformes promouvant une justice plus digne. À sa gauche, ils le sont également par les libéraux fervents comme Čičerin, mais aussi par de plus modérés qui l'accusent au mieux d'idéalisme, au pire de faire le lit de "l'Inquisition » en justifiant l'inclusion de dogmes religieux dans le domaine légal.

13 Vladimir Solov'ev fait en effet cavalier seul dans le camp des libéraux lorsqu'il publie Opravdanie dobra [La Justification du bien] ${ }^{2}$, puis Pravo i Nravstvennost' [Droit et morale] ${ }^{3}$. Comme son contemporain L. Tolstoj, le philosophe y exprime une défiance radicale visà-vis du droit de l'État, contre lequel il cherche des garde-fous. Se méfiant des excès du légalisme, il exclut, à l'inverse des positivistes, de fonder l'ordre légal sur l'État ; bien au contraire, il défend l'idée que toute norme, pour être légitime, doit être conforme à la morale chrétienne et se refuse à dissocier le droit de cette dernière. Cette posture lui permet de défendre comme les libéraux l'idée qu'il faut juger les hommes et non les crimes, que tout châtiment doit être digne et juste. Mais il ajoute que toute règle doit se conformer à un impératif de compassion et d'amour qui place les hommes sur un pied d'égalité devant le Tout-Puissant.

14 S'il est isolé en Russie, Solov'ev n'est cependant pas le seul philosophe à avoir défendu cette position. Il s'inscrit dans une tradition morale de la philosophie, identique à celle prônée par Georg Jellinek, qui a pleinement justifié la filiation de la loi à la morale. À la fin $d u X^{e}{ }^{e}$ siècle, cette pensée est considérée comme archaïque par les positivistes libéraux kantiens qui rejettent la morale dans la sphère personnelle du sujet (Čičerin, G.F. Šeršenevič). La morale relève pour eux du ressort de l'action individuelle et ne concerne 
pas l'ordre légal qui doit répondre à d'autres impératifs. Malheureusement, Frances Nethercott, faute de considérer pleinement ces distinctions, ne se pose pas la question de savoir si les positivistes libéraux ont réellement voulu dissocier le droit de la morale ou s'ils n'ont pas plutôt défendu une morale autre que celle de Solov'ev. Il y a en effet comme une dissymétrie dans le récit entre les thèses de Solov'ev qui fonde explicitement son engagement sur des principes moraux, et une frange de libéraux qui s'opposent à l'Empire sur autant de principes mais qui s'en défendent en mettant en avant une vision "positivée» du droit, par opposition à celle du gouvernement impérial. La confusion entre morale et droit revendiquée par Solov'ev était-elle réellement écartée par ses amis vestniki ? Leur mise en accusation de cette philosophie du droit ne masquait-elle pas leur volonté de promouvoir une morale émancipée de la morale chrétienne pour la fonder sur un pacte social et légal plutôt que sur l'amour chrétien? L'exposé de Frances Nethercott est sur ce point fort peu éclairant, voire confus.

L'ouvrage met finalement au-devant de la scène Vladimir Solov'ev, incarnation d'un libéralisme médian entre celui, radical, de Čičerin et le communautarisme slavophile, auxquels il s'est opposé violemment. Le philosophe figure ce libéralisme "à la russe ", méfiant envers la toute-puissance de l'État, tant sollicité dans la période contemporaine pour justifier les écarts des réformes russes vis-à-vis des théories libérales et de leurs dogmes individualistes abscons. À travers sa notoriété posthume dans la Russie postcommuniste, il devient finalement légitime de se demander si ses nouveaux adeptes ont véritablement endossé la thèse d'un droit raisonnable et conforme à la morale chrétienne, d'un droit de la piété et du partage - ou s'ils n'ont pas trouvé là une justification historique et nationale au droit russe, autrement dit une porte de sortie par le « haut » des apories libérales.

\section{NOTES}

2. SPb., 1897.

3. SPb., 1897. 